\title{
IMPLICATIONS FOR EVOLUTIONARY TRENDS FROM THE PAIRING FREQUENCIES AMONG GOLDEN-WINGED AND BLUE-WINGED WARBLERS AND THEIR HYBRIDS
}

\author{
John Confer ${ }^{1}$, Cody Porter $^{2}$, Kyle Aldinger ${ }^{3}$, Ronald Canterbury ${ }^{4}$, Jeffery Larkin ${ }^{5}$, and \\ Darin $\mathrm{McNeil}^{6}$ \\ ${ }^{1}$ Ithaca College \\ ${ }^{2}$ University of Wyoming \\ ${ }^{3}$ West Virginia University \\ ${ }^{4}$ University of Cincinnati \\ ${ }^{5}$ Indiana University of Pennsylvania \\ ${ }^{6}$ Pennsylvania State University
}

August 5, 2020

\begin{abstract}
Extensive range loss for the Golden-winged Warbler (Vermivora chrysoptera) has occurred in areas of intrusion by the Bluewinged Warbler (V. cyanoptera) potentially related to their close genetic relationship. We compiled data on social pairing from nine studies for 2,679 resident Vermivora to assess evolutionary divergence. Hybridization between pure phenotypes occurred with $1.2 \%$ of resident males for sympatric populations. Pairing success rates for Golden-winged Warblers was $83 \%$ and for Blue-winged Warblers was 77\%. Pairing success for the hybrid Brewster's Warbler was significantly lower from both species at $54 \%$, showing sexual selection against hybrids. Backcross frequencies for Golden-winged Warblers at $4.9 \%$ was significantly higher than for Blue-winged Warblers at $1.7 \%$. More frequent backcrossing by Golden-winged Warblers, which produces hybrid phenotypes, may contribute to the replacement of Golden-winged by Blue-winged Warblers. Reproductive isolation due to behavioral isolation plus sexual selection against hybrids was 0.966. Our analyses suggest that plumage differences are the main driving force for this strong isolation with reduced hybrid fitness contributing to a lesser degree. The major impact of plumage differences to reproductive isolation is compatible with genomic analyses (Toews et al. 2016), which showed the largest genetic difference between these phenotypes occurred with plumage genes. These phenotypes have maintained morphological, behavioral, and ecological differences during two centuries of hybridization. Our estimate of reproductive isolation supports recognition of these phenotypes as two species. The decline and extirpation of the Golden-winged Warbler in almost all areas of recent sympatry suggest that continued coexistence of both species will require eco-geographic isolation.
\end{abstract}

\section{Hosted file}

60613d1cd957784812ef70a6e07b3b277b793f2a.pdf available at https://authorea.com/users/315270/ articles/445623-implications-for-evolutionary-trends-from-the-pairing-frequencies-amonggolden-winged-and-blue-winged-warblers-and-their-hybrids

Table 1. Phenotype frequencies for resident Golden-winged and Blue-winged Warblers and their hybrids at each study area. 


\begin{tabular}{|c|c|c|c|c|c|c|c|c|c|c|c|c|c|}
\hline Sites & $\begin{array}{l}\text { Golden- } \\
\text { winged } \\
\text { Warbler }\end{array}$ & $\begin{array}{l}\text { Golden- } \\
\text { winged } \\
\text { Warbler }\end{array}$ & $\begin{array}{l}\text { Blue- } \\
\text { winged } \\
\text { Warbler }\end{array}$ & $\begin{array}{l}\text { Blue- } \\
\text { winged } \\
\text { Warbler }\end{array}$ & $\begin{array}{l}\text { Blue- } \\
\text { winged } \\
\text { Warbler }\end{array}$ & $\begin{array}{l}\text { Blue- } \\
\text { Winged } \\
\text { Warbler } \\
\\
\end{array}$ & $\begin{array}{l}\text { Percenta } \\
\text { GWWA1 } \\
\text { male } \\
\text { and } \\
\text { female } \\
\text { Golden- } \\
\text { winged } \\
\text { War- } \\
\text { bler } \\
\text { phe- } \\
\text { no- } \\
\text { types } \\
\text { as a } \\
\text { per- } \\
\text { cent- } \\
\text { age of } \\
\text { the } \\
\text { sum } \\
\text { of res- } \\
\text { ident } \\
\text { Golden- } \\
\text { winged } \\
\text { and } \\
\text { Blue- } \\
\text { winged } \\
\text { War- } \\
\text { bler } \\
\text { phenotyp }\end{array}$ & $\begin{array}{l}\text { gorercenta } \\
\text { male } \\
\text { and } \\
\text { female } \\
\text { Golden- } \\
\text { winged } \\
\text { War- } \\
\text { bler } \\
\text { phe- } \\
\text { no- } \\
\text { types } \\
\text { as a } \\
\text { per- } \\
\text { cent- } \\
\text { age of } \\
\text { the } \\
\text { sum } \\
\text { of res- } \\
\text { ident } \\
\text { Golden- } \\
\text { winged } \\
\text { and } \\
\text { Blue- } \\
\text { winged } \\
\text { War- } \\
\text { bler } \\
\text { po\$enotyp }\end{array}$ & 212Residen & $\begin{array}{l}\text { Brewster } \\
\text { Warbler }\end{array}$ & $\begin{array}{l}\text { r'Eawrence } \\
\text { Warbler }\end{array}$ & $\begin{array}{l}\text { elsawrence } \\
\text { Warbler }\end{array}$ & $\begin{array}{l}\text { elsaw] } \\
\text { Warl }\end{array}$ \\
\hline & male & female & female & male & female & & & male & male & & & & fema \\
\hline $\begin{array}{l}\text { Managed } \\
\text { forest, } \\
\text { high } \\
\text { eleva- } \\
\text { tion } \\
\text { PA }\end{array}$ & 137 & 100 & 100 & 4 & 2 & $97 \%$ & $97 \%$ & 12 & 12 & 6 & 6 & 0 & 0 \\
\hline $\begin{array}{l}\text { Pasture, } \\
\text { high } \\
\text { eleva- } \\
\text { tion } \\
\text { WV }\end{array}$ & 245 & 180 & 180 & 14 & 7 & $95 \%$ & $95 \%$ & 19 & 19 & 6 & 6 & 0 & 3 \\
\hline $\begin{array}{l}\text { Old } \\
\text { field, } \\
\text { low to } \\
\text { mid- } \\
\text { elevation } \\
\text { WV }\end{array}$ & 108 & 109 & 109 & 14 & 16 & $88 \%$ & $88 \%$ & 5 & 5 & 2 & 2 & 0 & 0 \\
\hline $\begin{array}{l}\text { Old } \\
\text { field, } \\
\text { north- } \\
\text { central } \\
\text { NY }\end{array}$ & 86 & 76 & 76 & 30 & 32 & $72 \%$ & $72 \%$ & 4 & 4 & 12 & 12 & 1 & 1 \\
\hline
\end{tabular}




\begin{tabular}{|c|c|c|c|c|c|c|c|c|c|c|c|c|c|}
\hline Sites & $\begin{array}{l}\text { Golden- } \\
\text { winged } \\
\text { Warbler }\end{array}$ & $\begin{array}{l}\text { Golden- } \\
\text { winged } \\
\text { Warbler }\end{array}$ & $\begin{array}{l}\text { Blue- } \\
\text { winged } \\
\text { Warbler }\end{array}$ & $\begin{array}{l}\text { Blue- } \\
\text { winged } \\
\text { Warbler }\end{array}$ & $\begin{array}{l}\text { Blue- } \\
\text { winged } \\
\text { Warbler }\end{array}$ & $\begin{array}{l}\text { Blue- } \\
\text { winged } \\
\text { Warbler }\end{array}$ & $\begin{array}{l}\text { Percenta } \\
\text { GWWA1 } \\
\text { male } \\
\text { and } \\
\text { female } \\
\text { Golden- } \\
\text { winged } \\
\text { War- } \\
\text { bler } \\
\text { phe- } \\
\text { no- } \\
\text { types } \\
\text { as a } \\
\text { per- } \\
\text { cent- } \\
\text { age of } \\
\text { the } \\
\text { sum } \\
\text { of res- } \\
\text { ident } \\
\text { Golden- } \\
\text { winged } \\
\text { and } \\
\text { Blue- } \\
\text { winged } \\
\text { War- } \\
\text { bler } \\
\text { phenotyr }\end{array}$ & $\begin{array}{l}\text { greercenta } \\
\text { male } \\
\text { and } \\
\text { female } \\
\text { Golden- } \\
\text { winged } \\
\text { War- } \\
\text { bler } \\
\text { phe- } \\
\text { no- } \\
\text { types } \\
\text { as a } \\
\text { per- } \\
\text { cent- } \\
\text { age of } \\
\text { the } \\
\text { sum } \\
\text { of res- } \\
\text { ident } \\
\text { Golden- } \\
\text { winged } \\
\text { and } \\
\text { Blue- } \\
\text { winged } \\
\text { War- } \\
\text { bler } \\
\text { opłenotyp }\end{array}$ & Brewste & $\begin{array}{l}\text { r'Brewster } \\
\text { Warbler }\end{array}$ & $\begin{array}{l}\text { r'Eawrence } \\
\text { Warbler }\end{array}$ & $\begin{array}{c}\text { elsawrenc } \\
\text { Warbler }\end{array}$ & $\begin{array}{l}\text { elsaw } \\
\text { War }\end{array}$ \\
\hline $\begin{array}{l}\text { Diverse } \\
\text { habi- } \\
\text { tats, s } \\
\text { NY, } \\
1998- \\
1999\end{array}$ & 62 & 37 & 37 & - & - & & & 13 & 13 & 1 & 1 & & \\
\hline $\begin{array}{l}\text { Diverse } \\
\text { habi- } \\
\text { tats, s } \\
\text { NY, } \\
2009- \\
2010\end{array}$ & 52 & 43 & 43 & 26 & 18 & $68 \%$ & $68 \%$ & 3 & 3 & 2 & 2 & 0 & 0 \\
\hline $\begin{array}{l}\text { Mine } \\
\text { lands, } \\
\text { mid- } \\
\text { elevation } \\
\text { WV }\end{array}$ & 298 & 267 & 267 & 246 & 185 & $57 \%$ & $57 \%$ & 27 & 27 & 21 & 21 & 2 & 7 \\
\hline $\begin{array}{l}\text { Old } \\
\text { field, } \\
\text { north- } \\
\text { central } \\
\text { MI }\end{array}$ & 26 & 13 & 13 & 23 & 15 & $51 \%$ & $51 \%$ & 3 & 3 & 0 & 0 & 0 & 0 \\
\hline
\end{tabular}




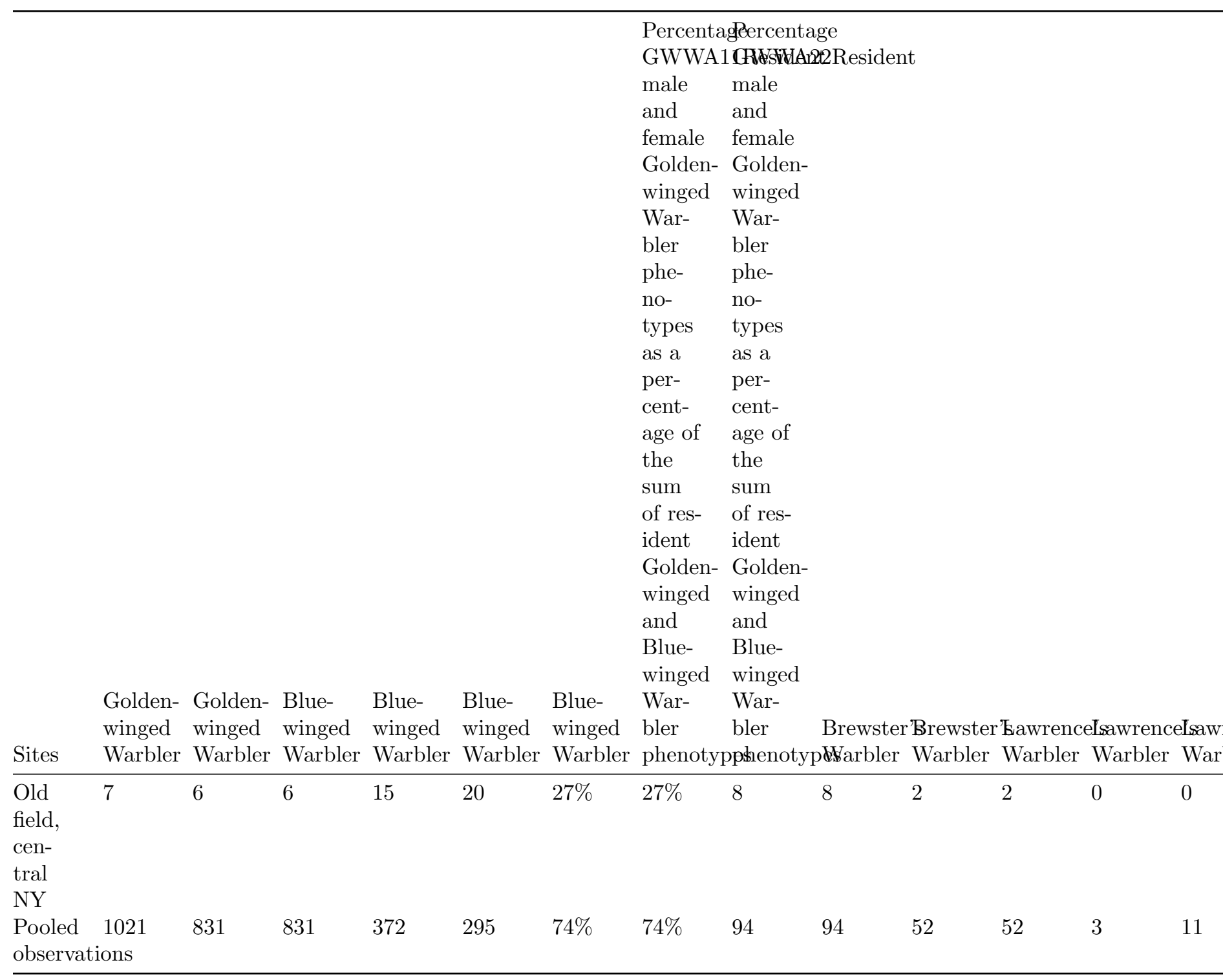

Table 2. Primary hybridization. Shown are the sum of paired individuals observed for all years at each study area and the sum of all study areas: A) the number of paired, male and female Golden-winged and Blue-winged warblers; B) the proportion of paired Golden-winged Warblers out of all paired Golden-winged and Blue-winged warblers; C) the proportion of primary hybrid pairs out of all pairs among Golden-winged and Blue-winged warblers, and the number of primary hybrid pairs.

\begin{tabular}{llll}
\hline Sites & $\begin{array}{l}\text { Number of Paired } \\
\text { Individuals }\end{array}$ & $\begin{array}{l}\text { Proportion of Paired } \\
\text { GWWA }\end{array}$ & Primary Hybridization \\
\hline $\begin{array}{l}\text { Managed forest, high } \\
\text { elevation, PA }\end{array}$ & GWWA BWWA & $98 \%$ & $3.0 \% 3$ \\
$\begin{array}{l}\text { Pasture, high elevation } \\
\text { WV }\end{array}$ & 35715 & $96 \%$ & $1.6 \% 3$
\end{tabular}




\begin{tabular}{|c|c|c|c|}
\hline Sites & $\begin{array}{l}\text { Number of Paired } \\
\text { Individuals }\end{array}$ & $\begin{array}{l}\text { Proportion of Paired } \\
\text { GWWA }\end{array}$ & Primary Hybridization \\
\hline $\begin{array}{l}\text { Old field, low to mid- } \\
\text { elevation WV }\end{array}$ & 21730 & $88 \%$ & $0.8 \% 1$ \\
\hline $\begin{array}{l}\text { Old field, north-central } \\
\text { NY }\end{array}$ & 16262 & $72 \%$ & $2.7 \% 3$ \\
\hline $\begin{array}{l}\text { Diverse habitats, s NY, } \\
\text { 1998-1999 }\end{array}$ & $74-$ & - & $4.0 \%^{1} 4$ \\
\hline $\begin{array}{l}\text { Diverse habitats, s NY, } \\
2009-2010\end{array}$ & 8637 & $70 \%$ & \\
\hline $\begin{array}{l}\text { Mine lands, } \\
\text { mid-elevation WV }\end{array}$ & 547370 & $60 \%$ & $0 \%$ \\
\hline $\begin{array}{l}\text { Old field, north-central } \\
\text { MI }\end{array}$ & 2629 & $47 \%$ & $0 \%$ \\
\hline Old field, central NY & 1335 & $27 \%$ & $0 \%$ \\
\hline Total & 1680583 & $73 \%$ & 14 \\
\hline Pooled Mean & & & $1.2 \%$ \\
\hline Mean of Study Areas & & & $1.5 \%$ \\
\hline
\end{tabular}

${ }^{1}$ Value derived from the sum of primary hybrid pairings of Golden-winged Warblers in 1998-'99 and primary hybrid pairings for Golden-winged and Blue-winged warblers in 2009-'10. Table 3. Pairing success rates for male Golden-winged, Blue-winged, and Brewster's warblers for nine studies and for the pooled values and for the mean of the nine studies.

\begin{tabular}{|c|c|c|c|c|c|c|c|c|}
\hline $\begin{array}{l}\text { Study } \\
\text { Areas }\end{array}$ & $\begin{array}{l}\text { Male } \\
\text { GWWA }\end{array}$ & $\begin{array}{l}\text { Male } \\
\text { GWWA }\end{array}$ & $\begin{array}{l}\text { Male } \\
\text { GWWA }\end{array}$ & $\begin{array}{l}\text { Male } \\
\text { BWWA }\end{array}$ & $\begin{array}{l}\text { Male } \\
\text { BWWA }\end{array}$ & $\begin{array}{l}\text { Male } \\
\text { BWWA }\end{array}$ & $\begin{array}{l}\text { Male } \\
\text { BRWA }\end{array}$ & $\begin{array}{l}\text { Male } \\
\text { BRWA }\end{array}$ \\
\hline & $n$ & $\%$ Paired & $n$ & $n$ & $\%$ Paired & $n$ & $n$ & $\%$ Pair \\
\hline $\begin{array}{l}\text { Managed } \\
\text { Forest, } \\
\text { high ele- } \\
\text { vation, } \\
\text { PA }\end{array}$ & 137 & $72 \%$ & 4 & 4 & $75 \%$ & 12 & 12 & $58 \%$ \\
\hline $\begin{array}{l}\text { Pasture, } \\
\text { high ele- } \\
\text { vation, } \\
\text { WV }\end{array}$ & 245 & $72 \%$ & 14 & 14 & $57 \%$ & 19 & 19 & $68 \%$ \\
\hline $\begin{array}{l}\text { Old } \\
\text { field, } \\
\text { low to } \\
\text { mid- el- } \\
\text { evation, } \\
\text { WV }\end{array}$ & 108 & $100 \%$ & 14 & 14 & $100 \%$ & 5 & 5 & $100 \%$ \\
\hline $\begin{array}{l}\text { Old } \\
\text { field, } \\
\text { north- } \\
\text { central } \\
\text { NY }\end{array}$ & 86 & $100 \%$ & 30 & 30 & $100 \%$ & 4 & 4 & $100 \%$ \\
\hline
\end{tabular}




\begin{tabular}{|c|c|c|c|c|c|c|c|c|}
\hline $\begin{array}{l}\text { Study } \\
\text { Areas }\end{array}$ & $\begin{array}{l}\text { Male } \\
\text { GWWA }\end{array}$ & $\begin{array}{l}\text { Male } \\
\text { GWWA }\end{array}$ & $\begin{array}{l}\text { Male } \\
\text { GWWA }\end{array}$ & $\begin{array}{l}\text { Male } \\
\text { BWWA }\end{array}$ & $\begin{array}{l}\text { Male } \\
\text { BWWA }\end{array}$ & $\begin{array}{l}\text { Male } \\
\text { BWWA }\end{array}$ & $\begin{array}{l}\text { Male } \\
\text { BRWA }\end{array}$ & $\begin{array}{l}\text { Male } \\
\text { BRWA }\end{array}$ \\
\hline $\begin{array}{l}\text { Diverse } \\
\text { habitats, s } \\
\text { NY, } \\
1998-1999\end{array}$ & 62 & $60 \%$ & & & & 13 & 13 & $8 \%$ \\
\hline $\begin{array}{l}\text { Diverse } \\
\text { habitats, s } \\
\text { NY, } \\
2009-2010\end{array}$ & 52 & $83 \%$ & 26 & 26 & $73 \%$ & 3 & 3 & $33 \%$ \\
\hline $\begin{array}{l}\text { Mine } \\
\text { lands, } \\
\text { mid- } \\
\text { elevation, } \\
\text { WV }\end{array}$ & 298 & $94 \%$ & 246 & 246 & $75 \%$ & 27 & 27 & $48 \%$ \\
\hline $\begin{array}{l}\text { Old } \\
\text { field, } \\
\text { north- } \\
\text { central, } \\
\text { MI }\end{array}$ & 26 & $50 \%$ & 23 & 23 & $61 \%$ & 3 & 3 & $33 \%$ \\
\hline $\begin{array}{l}\text { Old } \\
\text { field. } \\
\text { central } \\
\text { NY }\end{array}$ & 7 & $100 \%$ & 15 & 15 & $100 \%$ & 8 & 8 & $75 \%$ \\
\hline $\begin{array}{l}\text { Total } \\
\text { Pooled } \\
\text { Values }\end{array}$ & 1021 & $83 \%$ & 372 & 372 & $77 \%$ & 94 & 94 & $\begin{array}{l}51 \% \\
54 \%\end{array}$ \\
\hline $\begin{array}{l}\text { Study } \\
\text { Area } \\
\text { Means }\end{array}$ & & $81 \%$ & & & $80 \%$ & & & $58 \%$ \\
\hline
\end{tabular}

Table 4. Backcross Frequencies: Social pairs by male Golden-winged and Blue-winged warblers with hybrids.

\begin{tabular}{lllllllll}
\hline & GWWA & GWWA & GWWA & GWWA & BWWA & BWWA & BWWA & BWW \\
\hline Study Areas & & x BR & x LA & x BR + LA & & x BR & x LA & x BR \\
& $n$ & $\% n$ & $\% n$ & $\% n$ & $n$ & $\% n$ & $\% n$ & $\% n$ \\
Managed forest, high elevation PA & 98 & $5.1 \% 5$ & 0 & $5.1 \% 5$ & 3 & 0 & 0 & 0 \\
Pasture, high elevation WV & 177 & $4.0 \% 7$ & $0.6 \% 1$ & $4.5 \% 8$ & 8 & $12.5 \% 1$ & $25.0 \% 2$ & $37.5 \%$ \\
Old field, low to mid-elevation WV & 108 & $1.9 \% 2$ & 0 & $1.9 \% 2$ & 14 & 0 & 0 & 0 \\
Old field, north-central NY & 86 & $12.8 \% 11$ & $1.2 \% 1$ & $14.0 \% 12$ & 30 & 0 & 0 & 0 \\
Diverse habitats, sNY, 1998-1999 & 37 & 0 & 0 & 0 & - & - & - & - \\
Diverse habitats, sNY, 2009-2010 & 43 & $2.3 \% 1$ & 0 & $2.3 \% 1$ & 19 & $5.3 \% 1$ & 0 & $5.3 \% 1$ \\
Mine lands, mid-elevation WV & 280 & $5.0 \% 14$ & $2.5 \% 7$ & $7.5 \% 1$ & 185 & $1.6 \% 3$ & 0 & $1.6 \% 3$ \\
Old field, north-central MI & 13 & 0 & 0 & 0 & 14 & 0 & 0 & 0 \\
Old field, central NY & 7 & $8.6 \% 2$ & 0 & $28.6 \% 2$ & 15 & 0 & 0 & 0 \\
Total & 849 & 42 & 9 & 51 & 288 & 5 & 2 & 7 \\
Pooled Mean & & $4.9 \%$ & $1.1 \%$ & $6.1 \%$ & & $1.7 \%$ & $0.7 \%$ & $2.4 \%$ \\
Study Areas Mean & & $6.6 \%$ & $0.5 \%$ & $7.1 \%$ & & $2.4 \%$ & $3.1 \%$ & $5.5 \%$ \\
\hline
\end{tabular}




\begin{tabular}{lllllllll}
\hline & GWWA & GWWA & GWWA & GWWA & BWWA & BWWA & BWWA & BWWA \\
\hline Study Areas & & x BR & x LA & x BR + LA & & x BR & x LA & x BR + \\
& $n$ & $\% n$ & $\% n$ & $\% n$ & $n$ & $\% n$ & $\% n$ & $\% n$ \\
Managed forest, high elevation PA & 100 & $6.0 \% 6$ & 0 & $6.0 \% 6$ & 2 & 0 & 0 & 0 \\
Pasture, high elevation WV & 180 & $6.7 \% 12$ & 0 & $6.7 \% 12$ & 7 & $14.3 \% 1$ & $13.3 \% 2$ & $26.7 \% 4$ \\
Old field, low to mid-elevation WV & 109 & $3.7 \% 4$ & 0 & $3.7 \% 4$ & 16 & $6.3 \% 1$ & 0 & $3.3 \% 1$ \\
Old field, north-central NY & 76 & $1.3 \% 1$ & 0 & $1.3 \% 1$ & 32 & $6.3 \% 2$ & $1.6 \% 1$ & $4.5 \% 3$ \\
Diverse habitats, sNY, 1998-1999 & 37 & 0 & 0 & 0 & - & - & - & - \\
Diverse habitats, sNY, 2009-2010 & 43 & $2.3 \% 1$ & 0 & $2.3 \% 1$ & 18 & 0 & 0 & $2.7 \% 3$ \\
Mine lands, mid-elevation WV & 267 & $3.0 \% 8$ & 0 & $3.0 \% 8$ & 185 & $0.5 \% 1$ & $1.1 \% 4$ & $1.6 \% 1$ \\
Old field, north-central MI & 13 & 0 & 0 & 0 & 15 & $6.7 \% 1$ & 0 & $3.4 \% 1$ \\
Old field, central NY & 6 & $16.7 \% 1$ & 0 & $16.7 \% 1$ & 20 & $25.0 \% 5$ & 0 & $14.3 \% 5$ \\
Total & 831 & 33 & 0 & 33 & 295 & 11 & 3 & 14 \\
Pooled Mean & & $4.0 \%$ & 0 & $4.0 \%$ & & $3.7 \%$ & $1.0 \%$ & $4.7 \%$ \\
Study Areas Mean & & $4.4 \%$ & 0 & $4.4 \%$ & & $7.4 \%$ & $0.45 \%$ & $7.9 \%$ \\
\hline
\end{tabular}

Table 5. Backcross frequencies. Social pairs by female Golden-winged and Blue-winged warblers with hybrids.

Table 6. Backcross Frequencies: Social pairs by male and female Golden-winged and Blue-winged warblers with hybrids.

\begin{tabular}{lllllllll}
\hline & GWWA & GWWA & GWWA & GWWA & BWWA & BWWA & BWWA & BWWA \\
\hline Study Areas & & x BR & x LA & x BR + LA & & x BR & x LA & x BR + \\
& $n$ & $\% n$ & $\% n$ & $\% n$ & $n$ & $\% n$ & $\% n$ & $\% n$ \\
Managed forest, high elevation PA & 198 & $5.6 \% 11$ & 0 & $5.6 \% 11$ & 5 & 0 & 0 & 0 \\
Pasture, high elevation WV & 357 & $5.3 \% 19$ & $0.3 \% 1$ & $5.6 \% 20$ & 15 & $13.3 \% 2$ & $13.3 \% 2$ & $26.7 \%$ \\
Old field, low to mid-elevation WV & 217 & $2.8 \% 6$ & 0 & $2.8 \% 6$ & 30 & $3.3 \% 1$ & 0 & $3.3 \% 1$ \\
Old field, north-central NY & 162 & $7.4 \% 12$ & $0.6 \% 1$ & $8.0 \% 13$ & 62 & $3.2 \% 2$ & $1.6 \% 1$ & $4.5 \% 3$ \\
Diverse habitats, sNY, 1998-1999 & 74 & 0 & 0 & 0 & - & - & - & - \\
Diverse habitats, sNY, 2009-2010 & 86 & $2.3 \% 2$ & 0 & $2.3 \% 2$ & 37 & $2.7 \% 3$ & 0 & $2.7 \% 3$ \\
Mine lands, mid-elevation WV & 547 & $4.0 \% 22$ & $1.3 \% 7$ & $5.3 \% 29$ & 370 & $1.1 \% 4$ & $1.1 \% 4$ & $1.6 \% 1$ \\
Old field, north-central MI & 26 & 0 & 0 & 0 & 29 & $3.4 \% 1$ & 0 & $3.4 \% 1$ \\
Old field, central NY & 13 & $23.1 \% 3$ & 0 & $23.1 \% 3$ & 35 & $14.3 \% 5$ & 0 & $14.3 \% 5$ \\
Total & 1680 & 75 & 9 & 84 & 583 & 16 & 5 & 21 \\
Pooled Mean & & $4.5 \%$ & $0.54 \%$ & $5.0 \%$ & & $2.7 \%$ & $0.86 \%$ & $3.6 \%$ \\
Study Areas Mean & & $5.6 \%$ & $0.24 \%$ & $5.8 \%$ & & $5.2 \%$ & $1.9 \%$ & $7.1 \%$ \\
\hline
\end{tabular}




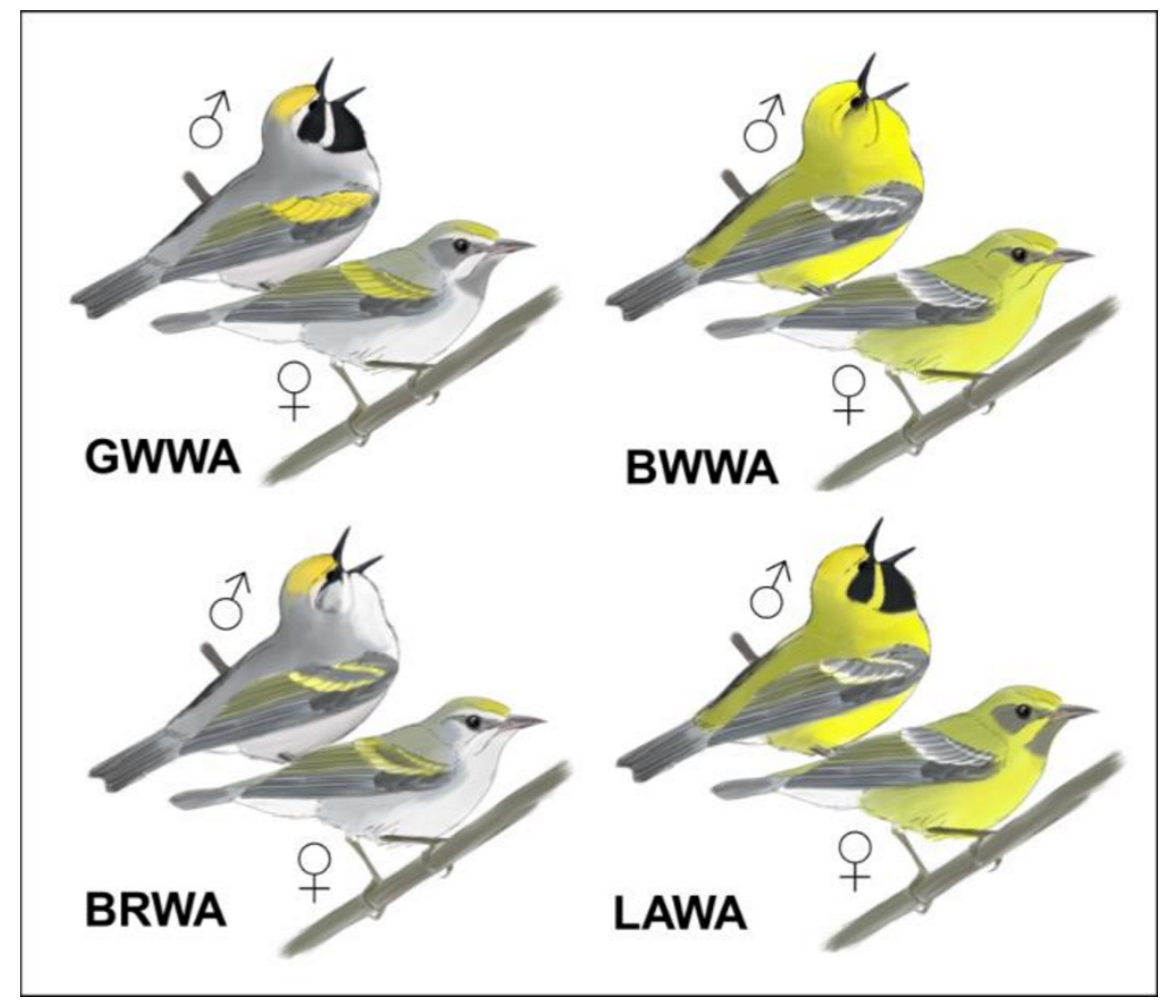

Figure 1. Vermivora spp. phenotypes considered in this study: Golden-winged Warbler (V. chrysoptera ; GWWA), Blue-winged Warbler (V. cyanoptera ; BWWA), 'Brewster's' Warbler (hybrid; BRWA) and 'Lawrence's' Warbler (hybrid' LAWA). Males () and females () are both shown. 


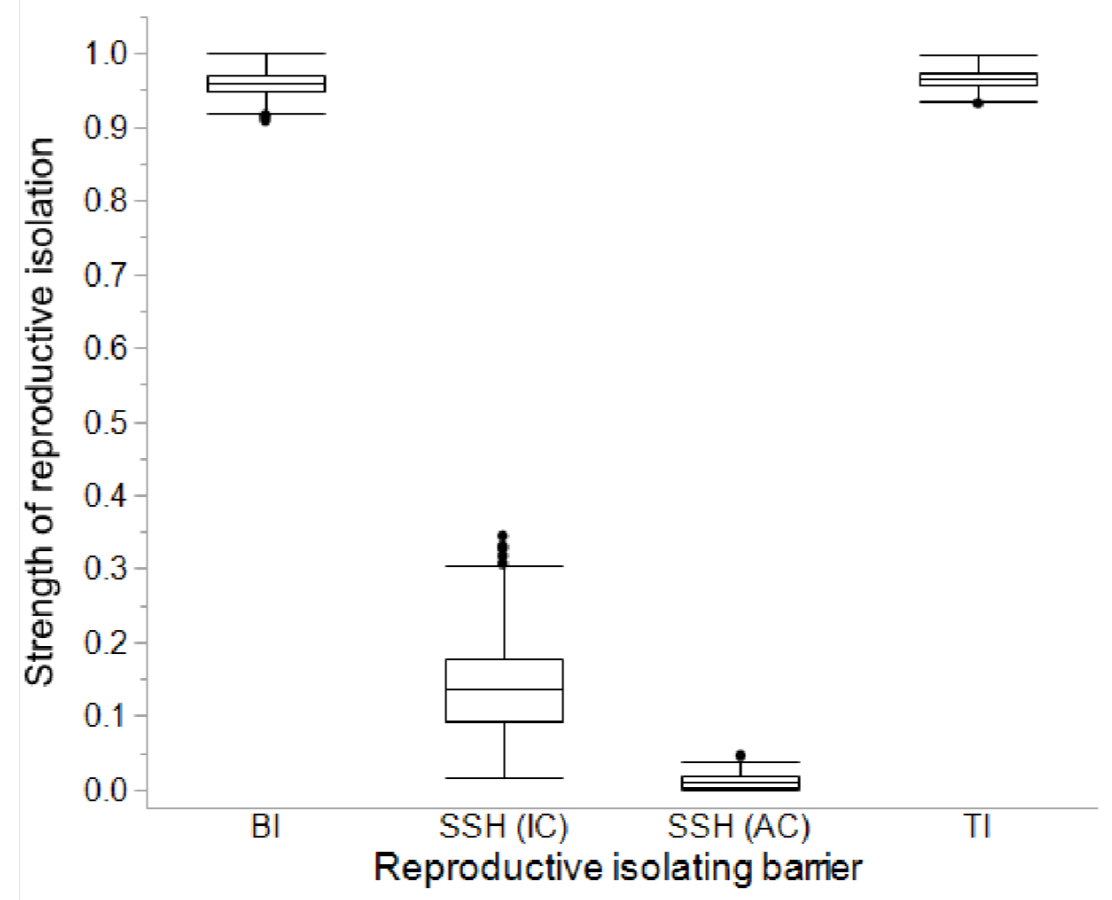

Figure 2. Box and whisker plot of the strength of reproductive isolating barriers (1000 bootstrapped averages). Abbreviations denote the following: behavioral isolation (BI), individual component of sexual selection against hybrids ( $\mathrm{SSH}(\mathrm{IC})$ ), absolute contribution of sexual selection against hybrids ( $\mathrm{SSH}(\mathrm{AC})$ ), and total reproductive isolation (TI). 


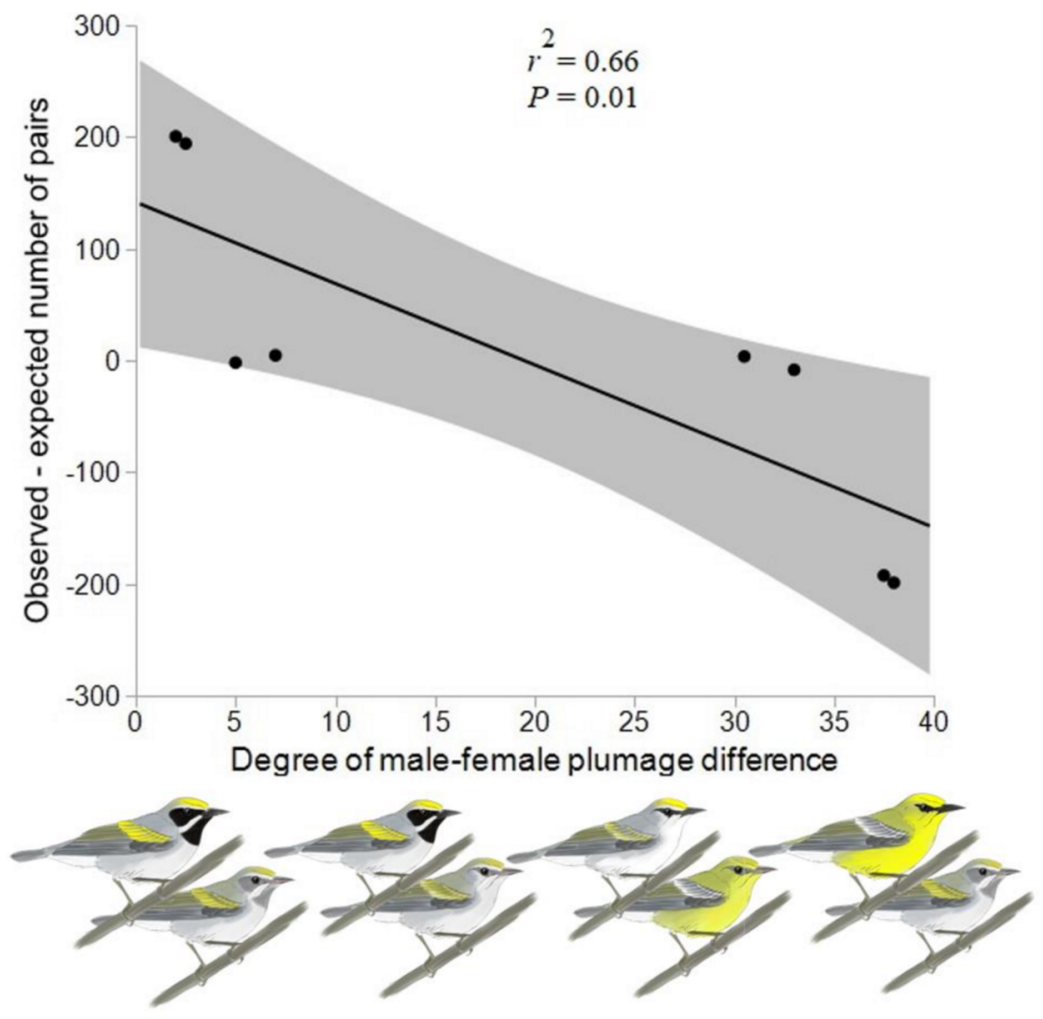

Figure 3. As the difference in plumage between males and females increases, the likelihood of mating (observed number of pairs - expected number of pairs due to random chance) decreases. Plumage was quantified by scoring 11 plumage patches on males and females of each phenotypic class (see Supporting Information for plumage scoring criteria). Below the $x$-axis are representative illustrations of the different pair types (top row: males, bottom row: females), depicting the male-female plumage divergence with increasing values of $\mathrm{x}$.

Appendix 1. A plumage scoring criterion. We modified the method of Toews et al. (2016) to rank Vermivora spp. plumage with the exception of three edits (denoted using asterisks within table). Intermediate throat pigmentation ("Thr.") between a Golden-winged Warbler (Thr. =0) and Blue-winged Warbler (Thr = 2) should resemble a Brewster's Warbler (i.e ., white in color) rather than that of a second-year male Golden-winged Warbler as in Teows et al. (2016). Additionally, breast plumage color ("Breast") and belly plumage color ("Belly") for adult male Blue-winged Warblers are bright yellow (rather than yellow-green) so those plumage categories were extended (from 4- to 5). All other plumage scoring criteria were identical to those used by Toews et al. (2016): wing bar width ("W. Bar Wd."), wing bar color ("W. Bar. Col."), nape color ("Nape"), back color ("Back"), rump color ("Rump"), auricular pigmentation pattern ("Auric."), supercillium color ("Super."), and malar color ("Malar"). A male Blue-winged Warbler with typical plumage would score 40 using this protocol whereas a Golden-winged Warbler would score 0.

\begin{tabular}{lllll}
\hline Score & Thr. & W. Bar Wd. & W. Bar Col. & Nape \\
\hline 0 & black/gray & broad, confluent & yellow & gray or white \\
1 & white* $^{*}$ & broad, well-separated & yellow with pale base & Gray or white with some yellow or yellow-gr \\
2 & yellow & broad, less separated & white and yellow $(50 / 50)$ & mixed gray and green \\
3 & - & narrow, well-separated & white with pronounced yellow & yellow-green with gray
\end{tabular}




\begin{tabular}{lllll}
\hline Score & Thr. & W. Bar Wd. & W. Bar Col. & Nape \\
\hline 4 & - & - & yellow edging & yellow-green \\
5 & - & - & white & - \\
\hline
\end{tabular}

Acknowledgements.

The data for the analyses of this manuscript came from studies that were published previously. This compilation was not directly supported by any grant. The 47 years of field studies required the effort of a large number of dedicated and much appreciated field assistants. J Gillis, W MacCabe, L. Stenzler, T. Schat reviewed versions of the manuscript. The senior author thanks his spouse for 40 years of support during studies of Golden-winged and Blue-winged warblers.

\section{Contributions}

J. Confer initiated the effort to pool several studies in order to create a data base sufficient to analyze uncommon but significant events with hybrids. C. Porter was responsible for the analyses of reproductive isolation. D. McNeil was responsible for all illustrations and statistical analyses relating phenotype differences to frequency of mating. All authors contributed to the analyses, data interpretation, and manuscript writing. All authors contributed to the sometimes lengthy and vigorously debated revisions of the manuscript. All authors approved this version as submitted for review. 